ised and karyorrhectic nuclei, differences in the appearance of individual nuclei within the same erythroblast, and focal loss of nuclear membrane material. Mitochondrial abnormalities, autophagic vacuoles, extensive myelinisation of the nuclear membrane, and the presence of intracytoplasmic myelin figures have also been reported.

CDA type III has been described in only a few families and individuals. ${ }^{134679}$ Sandstrom $e t a l^{\beta}$ in 1994 in an extensive investigation in a known family with CDA type III $^{5}$ observed a high prevalence of monoclonal gammopathy of undetermined significance and multiple myeloma. The authors speculated that genes for the development of monoclonal gammopathy/myeloma and CDA type III may be linked. Prior to this, Byrnes et al in 1980 described an individual who had been treated by radiotherapy for Hodgkin's disease and was found to have abnormalities of erythroblasts on a bone marrow aspirate. The abnormalities were initially interpreted as being radiotherapy induced but review of a bone marrow aspirate taken 18 years earlier revealed identical changes in the red cell series and a diagnosis of CDA type III was established.

In conclusion, we present bone marrow aspirate findings and ultrastructural features of erythroblasts in a case of CDA type III. The patient developed a malignant $T$ cell lymphoma with cutaneous and widespread nodal involvement. This case, in conjunction with previous studies, ${ }^{34}$ suggests that there may be an increased risk of development of lymphoproliferative disease in CDA type III. The mechanism of this association is not clear but it may be that genes for the development of $\frac{\rho}{5}$ CDA type III are linked to those which control 0 lymphoid proliferation or development.

1 Wolff JA, Von Hofe FH. Familial erythroid multinuclearity. Blood 1951;6:1274-83.

2 Heimpel H, Wendt F. Congenital dyserythropoietic anaemia with karyorrhexis and multinuclearity of erythroblasts. with karyorrhexis and multin

3 Sandstrom H, Wahlin A, Eriksson M, Bergstrom I, Wickramasinghe SN. Intravascular haemolysis and increased $\mathbb{\Phi}$ prevalence of myeloma and monoclonal gammopathy in congenital dyserythropoietic anaemia, type III. Eur $\mathcal{F} \mathrm{Hae}-\mathrm{n}$ matol 1994;52:42-6.

4 Byrnes RK, Dhru R, Brady AM, Galen WP, Hopper B. $\vec{O}$ Congenital dyserythropoietic anaemia in treated Hodgkin's disease [letter]. Hum Pathol 1980;5:485-6.

Wickramasinghe SN, Wahlin A, Anstee D, Parsons SF, Stopps G, Bergstrom I, et al. Observations on two members of the Swedish family with congenital dyseryth-? ropoietic anaemia, type III. Eur f Haematol 1993;50:213-21. I

6 Bergstrom I, Jacobsson L. Hereditary benign erythroreticulosis. Blood 1962;19:296-303.

7 Goudsmit R, Beckers D, DeBruijne JL, Engelfriet CP, James 9 J, Morselt AFW, et al. Congenital dyserythropoietic N $\mathrm{J}$, Morselt AFW, et al. Congenital dyserythro

8 Wickramasinghe SN, Parry TE, Williams C, Bond AN, 을 Hughes $M$, Crook S. A new case of congenital dyserythropoietic anaemia, type III: Studies of the cell cycle distribu- $c$ tion and ultrastructure of erythroblasts and of nucleic acid synthesis in marrow cells. $\mathcal{F}$ Clin Pathol 1982;35:1103-9.

9 Choudhry VP, Saraya AK, Kasturi J, Rath PK. Congenital dyserythropoietic anaemias: splenectomy as a mode of therapy. Acta Haematol 1981;66:195-201.

\title{
Duodeno-jejunal adenocarcinoma as a first presentation of coeliac disease
}

\author{
D J L MacGowan, D O'B Hourihane, W A Tanner, C O'Morain
}

\begin{abstract}
Department of Gastroenterology, Meath/Adelaide and St James's Hospitals, Trinity College, Dublin, Ireland C O'Morain

Department of Surgery W A Tanner

Department of Pathology

D J L MacGowan

D O'B Hourihane

Correspondence to: Professor Colm O'Morain, Department of Gastroenterology, The Meath Hospital Heytesbury Street, Dublin 2,

Ireland.

Abstract

Long standing coeliac disease is associated with an increased risk of malignancy, not only of intestinal lymphoma but also small intestinal adenocarcinoma. Two patients whose initial presentation was adenocarcinoma of the small bowel, but who were subsequently found to have coeliac disease after Whipple's resection, are described. The diagnosis was made early in the postoperative period in the first patient after close histological examination of the tumour-free mucosal margins. This patient was placed on a gluten-free diet and had an uncomplicated postoperative recovery with rapid weight gain. Diagnosis and dietary intervention in the second patient was very delayed and resulted in the development of severe malabsorption and weight loss. This illustrates the importance of ruling out coeliac disease prior to surgery in patients with small intestinal malignancies.

$(\mathcal{C}$ Clin Pathol 1996;49:602-604)
\end{abstract}

Keywords: small bowel, adenocarcinoma, coeliac disease.
Small intestinal adenocarcinoma is 82 times more common in patients with coeliac disease $\frac{D}{0}$ than in the normal population, making it as common a tumour as colon cancer in these patients. ${ }^{1}$ o This relation has been reported in a total of $40 \mathrm{~N}$ patients. ${ }^{1-8}$ In the 19 patients for whom adequate information is available, ${ }^{235-8}$ the presentation of 0 malignancy was preceded by symptoms ofo malabsorption and usually a longstanding history $₫$ of coeliac disease. This is therefore the first report ${ }^{+}$ describing two patients in whom the diagnosis of small bowel adenocarcinoma was not preceded $\stackrel{\circ}{\mathrm{\sigma}}$ by a history of malabsorption. Both patients $\stackrel{\sim}{\mathbb{D}}$ developed symptoms of malabsorption after sur- $\frac{\alpha}{9}$ gery, raising a possible mechanism of latent coeliac disease being unmasked by surgery. Latento̊ coeliac disease (high intraepithelial lymphocyte count with positive $\alpha$-gliadin antibodies) is ano을 increasingly recognised entity, ${ }^{9}$ but a second factor seems to drive this minimal enteropathy from latent to clinically overt disease. Following gastrectomy or upper gastrointestinal surgery, the bowel may be transiently hyperpermeable on reintroduction of oral intake and the intraluminal 
digestion of gluten may be impaired. Consequently, there may be an acute surge in gluten challenge resulting in overt disease. ${ }^{9}$

\section{Case reports}

PATIENT 1

A 59 year old woman presented with a six month history of projectile vomiting, postprandial fullness and $20 \mathrm{~kg}$ weight loss. Barium meal and follow through revealed retained gastric fluid and dilated loops of small bowel in the upper abdomen, suggestive of a partially obstructive lesion. She proceeded to laparotomy, where a $5 \times 3 \mathrm{~cm}$ jejunal tumour was seen close to the duodeno-jejunal flexure with enlarged mesenteric nodes. A Whipple's resection was performed. Histopathology confirmed a stricturing adenocarcinoma with clear resection margins, but a small focus of metastatic deposit in one of three regional lymph nodes. The mucosa not involved by tumour demonstrated severe villous blunting with a prominent mononuclear infiltrate in the lamina propria (fig 1A). Alpha-gliadin antibodies were also elevated at $2.6 \mathrm{IU} / 1$ (normal $=1.27-1.43$ $\mathrm{IU} / \mathrm{l}$ ) and folate levels reduced to $0.5 \mathrm{ng} / \mathrm{l}$.
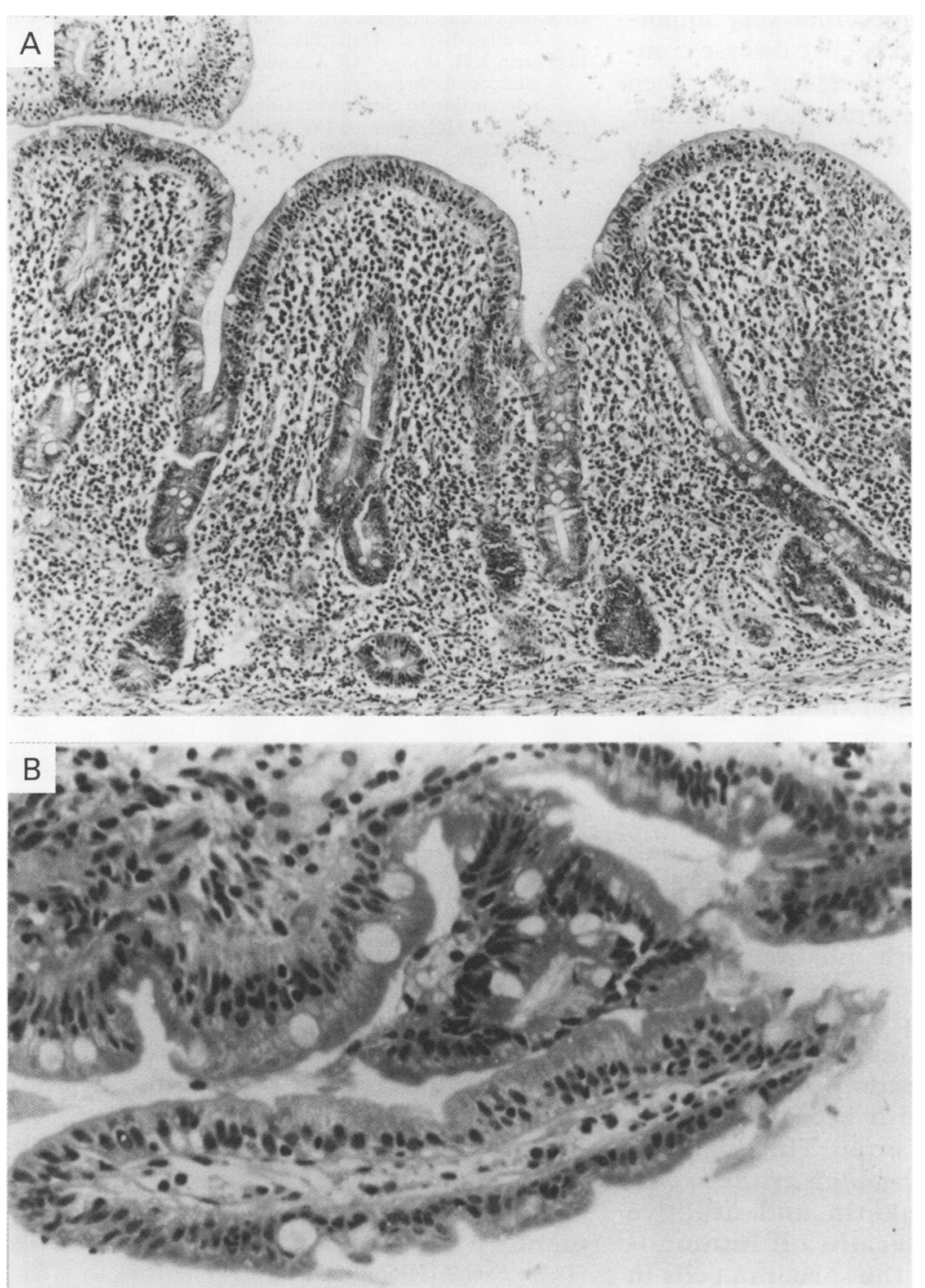

Figure 1 Mucosa from the distal surgical specimen in $(A)$ shows increased intraepithelial lymphocytes with inflammatory cells in the lamina propria and short, blunted villi. (B) The jejunal mucosa reverses to a normal pattern after the introduction of a strict gluten-free diet.
Postoperatively, the patient was commenced on a gluten-free diet and recovered rapidly. Her weight increased steadily by over $20 \mathrm{~kg}$. Repeat endoscopic small bowel biopsy two months later showed reversal of the duodenal mucosa to a normal pattern (fig 1B). A gluten challenge resulted in abdominal pain and diarrhoea within 10 days and repeat duodenal biopsy confirmed coeliac disease. She remained well for five years until local and distal recurrence developed and she died from intestinal obstruction and perforation.

\section{PATIENT 2}

A 51 year old woman presented with a four month history of epigastric pain and $6 \mathrm{~kg}$ weight loss. Her bowel habit was normal and she was not anaemic. A polyp of $7 \mathrm{~cm}$ was seen in the second part of the duodenum at endoscopy. Biopsy revealed an invasive adenocarcinoma. A computed tomography (CT) scan of the abdomen showed no evidence of metastases. An uncomplicated Whipple's procedure followed and histopathology showed that all of the operative margins and accompanying local lymph nodes were free of tumour. Postoperatively, the patient initially did well until she recommenced oral intake when she developed abdominal cramps and loose, watery diarrhoea. This pattern persisted over the next three months and her weight fell by $25 \mathrm{~kg}$, with profound resulting cachexia despite documented daily caloric intakes of over $2000 \mathrm{kcal}$. Repeated CT scans of the abdomen showed no abnormalities apart from notable fatty infiltration of the liver. Jejunal biopsy was then performed endoscopically and this showed total villous atrophy with marked plasma cell infiltration. The tumour-free mucosal margins of the original were then re-inspected for evidence of coeliac disease. There was subtotal villous atrophy along with an intense mononuclear inflammatory infiltrate in the lamina propria. The original diagnosis of coeliac disease was also supported by elevated measurements of both the serum $\alpha$-gliadin and antiendomysial antibodies. On introduction of a gluten-free diet, her bowel habit returned to normal within one month and her weight had increased by $10 \mathrm{~kg}$. Although she remains well, she has not been strictly adhering to her diet. This is reflected in her most current jejunal biopsy which is improved but not completely returned to normal and her persistently elevated anti-endomysial antibodies.

\section{Discussion}

Small intestinal adenocarcinomas are rare tumours. The largest series is of 209 cases over 30 years in Saskatchewan, giving an annual incidence rate of 0.7 and 0.6 per 100000 for men and women, respectively. Swinson et al documented an 82-fold increase in incidence in the coeliac population when they found 19 invasive small intestinal adenocarcinomas out of 259 histologically confirmed malignancies in 235 patients with histologically confirmed coeliac disease. Lymphoma was a much more frequently complicating neoplasm and it has been shown that over the age of 50 years, there is a 1 
in 10 risk of intestinal lymphoma in a newly diagnosed patient with coeliac disease. ${ }^{10} \mathrm{Ex}$ actly how and why coeliac disease is associated with an increased risk of lymphomatous and carcinomatous change remains unknown. Several explanations have been put forward. The mucosal damage in coeliac disease may make the small intestine more permeable to environmental carcinogens which, with associated malabsorptive vitamin A deficiency and increased vulnerability to oxidative injury, would enhance carcinogenic change. Known predisposing factors for the development of small intestinal adenocarcinoma include Crohn's disease, adenomatous polyps and PeutzJeugher's syndrome. ${ }^{4}$ Perzin et al ${ }^{11}$ reported that 23 of 51 cases of small intestinal adenocarcinoma had evidence of adenoma and carcinoma in the same lesion. Survival is dependent on the presence or absence of nodal involvement at presentation. Five year survival with node negative disease is $68 \%$, while the overall figure varies from 15 to $20 \% .{ }^{12}$ Thus, as the prognosis is not uniformly poor and some patients are potentially curable by total resection (as in patient 2 in the present paper), limiting postoperative morbidity and recognising potential complications becomes very important. Clinically unmasked coeliac disease complicating Whipple's resection has not been described before and these two cases illustrate the importance of early diagnosis and dietary intervention prior to surgery. Previous cases of failure to thrive and weight loss following Whipple's resection may be simply put down to the effects of small bowel resection, pancreatic insufficiency, tumour progression, or early recurrence. Underlying coeliac disease should also be considered and the tumour-free mucosal margins carefully inspected.

1 Swinson CM, Slavin G, Coles EC, Booth CC. Coeliac disease and malignancy. Lancet 1983;i:111-15.

2 Holmes GKT, Dunn GI, Cockel R, Brookes VS. Adenocarcinoma of the upper small bowel complicating coeliac disease. Gut 1980;21:1010-16.

3 Selby WS, Gallagher ND. Malignancy in a 19 year experience of adult Coeliac disease. Dig Dis Sci 1979, 24:684-8.

4 Lioe TF, Biggart JD. Primary adenocarcinoma of the jejunum and ileum: Clincopathological review of 25 cases. f Clin Pathol 1990;43:533-6.

5 Farrell DJ, Shrimankar J, Griffin SM. Duodenal adenocarcinoma complicating coeliac disease. Histopathology 1991; 19:285-7.

6 Straker RJ, Gunasekaran S, Brady PG. Adenocarcinoma of the jejunum in association with Coeliac sprue. $\mathcal{F}$ Clin Gas troenterol 1989;11:320-3.

7 Marignani $M$, Levin MF, Bach DB, Sinha $R$. Coeliac disease complicated by adenocarcinoma. Resident's case of the month. Can Assoc Radiol f 1993;44:481-4.

8 Pelli MA, Cavalletti ML, Bassotti G, Ribacchi F, Morelli A. Adenocarcinoma of the duodenal bulb in a young Coeliac woman. Ital f Gastroenterol 1993;25:121-2.

9 Ferguson A, Arranz E, O'Mahony S. Clinical and pathological spectrum of Coeliac disease - active, silent, latent, cal spectrum of Coeliac disea

10 Cooper BT, Holmes GK Cooke WT Lymphoma risk in Coeliac disease of later life. Digestion 1982;23:89-92.

11 Perzin KH, Bridge MF. Adenomas of the small intestine: A clinicopathological review of 51 cases and a study of their relationship to carcinoma. Cancer 1981;48:799-819.

12 Barclay THC, Schapira DV. Malignant tumours of the small intestine Cancer 1983;51:878-81.

\title{
Mixed low grade and high grade endometrial stromal sarcoma of uterus: differences on immunohistochemistry and chromosome in situ hybridisation
}

\author{
A N-Y Cheung, W-F Ng, L-P Chung, U-S Khoo
}

\section{Department of Pathology, University of Hong Kong, \\ Hong Kong \\ Correspondence to: Dr Annie Cheung, Department of Pathology, University of Hong Kong, Queen Mary Hospital, Pokfulam Road, \\ Hong Kong.}

Accepted for publication Accepted for publict

\section{Abstract}

A case of a 64 year old woman with a tumour of the uterus is reported. The patient presented with postmenopausal bleeding and subsequently underwent total hysterectomy and bilateral salpingooophorectomy. Sections of the tumour showed a low grade endometrial stromal sarcoma coexisting with areas consistent with high grade sarcoma. The sarcoma cells, in both the low and high grade areas, were positive for vimentin and negative for desmin and cytokeratin on immunohistochemistry. While the sarcoma cells in the low grade region showed immunoreactivity for oestrogen and progestogen receptors, those in the high grade region did not. Using chromosome in situ hybridisation, the low grade portion of the sarcoma was diploid for chromosomes $X$, 11,12 , and 17, whereas the more anaplastic areas were aneuploid for these chromosomes. This case may represent an example of high grade endometrial stromal sarcoma arising by dedifferentiation from a low grade stromal sarcoma. Adequate sampling is important in identifying such anaplastic changes as the origin of the tumour will affect patient management.

(F Clin Pathol 1996;49:604-607)

Keywords: endometrial stromal sarcoma, dedifferentiation. 